\title{
Multiple observations of cavitation cluster dynamics close to an ultrasonic horn tip.
}

Peter R. Birkin, Douglas G. Offin, Christopher J. B. Vian

School of Chemistry, University of Southampton, Southampton UK SO17 1BJ. Email:prb2@soton.ac.uk

Please cite this paper as:

Journal of the Acoustical Society of America, 2011 , (130) 3379-3388

The publisher's version of this paper is available here:

http://dx.doi.org/10.1121/1.3650536

Related articles by Dr Peter Birkin can be found below:

D. G. Offin, P. R.Birkin, T. G. Leighton, P. J. Joseph, (2006).

Acoustoelectrochemistry: The application of sound to electrochemical processes.

Proceedings of the Institute of Acoustics 28, (1), 821-829.

C. J. B. Vian, P. R. Birkin, T. G. Leighton, (2009). Opto-Isolation of Electrochemical Systems in Cavitation Environments. Analytical Chemistry 81, (12), 5064-5069. (doi:10.1021/ac802561k).

C. J. B. Vian, P. R. Birkin, T. C. Leighton, (2010). Cluster Collapse in a Cylindrical Cell: Correlating Multibubble Sonoluminescence, Acoustic Pressure, and Erosion. Journal of Physical Chemistry C , 114, (39), 16416-16425. (doi:10.1021/jp1027977). 
Birkin et al.

\title{
Multiple observations of cavitation cluster dynamics close to an ultrasonic horn tip
}

\author{
Peter R. Birkin*, Douglas G. Offin, Christopher J. B. Vian \\ School of Chemistry, University of Southampton, Southampton, SO17 1BJ, UK. \\ E-mail:prb2@soton.ac.uk \\ Timothy G. Leighton \\ Institute of Sound and Vibration Research , University of Southampton, Southampton, \\ SO17 1BJ, UK.
}

Running title: Clusters observed with multiple sensors

PACS Numbers: 43.35.Ei, 43.35.Ty, 43.25.Yw, 43.25.Ts, 43.35.Vz

Keywords: clusters, erosion, luminescence, imaging

* author for correspondence 
Birkin et al.

\begin{abstract}
Bubble dynamics in water close to the tip of an ultrasonic horn $(\sim 23 \mathrm{kHz}, 3 \mathrm{~mm}$ diameter $)$ have been studied using electrochemistry, luminescence, acoustics, light scattering and high-speed imaging. It is found that, under the conditions employed, a large bubble cluster ( $\sim 1.5 \mathrm{~mm}$ radius $)$ exists at the tip of the horn. This cluster collapses periodically every 3 to 4 cycles of the fundamental frequency of the horn. Following the collapse of the cluster, a short-lived cloud of small (10's of microns in diameter) bubbles were observed in the solution. Large amplitude pressure emissions are also recorded which correlate temporally with the cluster collapse. Bursts of surface erosion (measured in real time using an electrochemical technique) and multibubble sonoluminescence (MBSL) emission both also occur at a sub harmonic of the fundamental frequency of the horn and are temporally correlated with the bubble cluster collapse and the associated pressure wave emission.
\end{abstract}


Birkin et al.

\section{Introduction}

The ultrasonic horn is one of the most common commercial ultrasonic devices, and yet its mode of operation is surprisingly complex. Through the application of an approximately sinusoidal continuous-wave (or sometimes tone-burst) voltage (of centre frequency $f_{0}$ usually in the region of $20-30 \mathrm{kHz}$ ) to the piezoelectric stack in the shaft of the horn, the tip of the horn undergoes an approximately sinusoidal oscillation of a few 10's of microns at $f_{0}$. The tip being immersed in a liquid, and the wavelength in the liquid at $f_{0}$ being much larger than the tip diameter, the direct sound field amplitude falls off rapidly with distance from the tip (as a dipole if the tip is only shallow, and as a monopole if it is very deep, which is rare). Even in the absence of cavitation, the overall sound field in the liquid itself is a combination of this direct field and that generated by the reverberation and reflections from walls of the vessel which contain the liquid ${ }^{1,2}$. When cavitation is modelled in this environment, the vast majority of simulations assume a single bubble in an infinite body of liquid driven by a sound field at $f_{0}$. If the driving pressure fluctuations are great enough to exceed the Blake threshold (usually around atmospheric pressure at these frequencies under normal conditions in water) microscopic pre-existing bubbles within the liquid can expand rapidly in the rarefaction portion of a sound wave. If this expansion is sufficiently large, the subsequent bubble collapse is dominated by inertial forces within the liquid and has been termed 'inertial cavitation' (also known as 'transient cavitation') ${ }^{3,4}$. In contrast, during 'non inertial cavitation' the collapse is dominated by the pressure within the gas phase of the bubble interior ${ }^{5-7}$. The fate of a particular bubble is determined by the local conditions of frequency, pressure amplitude and solution parameters ${ }^{3}{ }^{8}$, and its initial size, and these define the threshold conditions for generating inertial cavitation. The most common usage of ultrasonic horns is for them to generate $20-30 \mathrm{kHz}$ continuous-wave fields into water or aqueous solution 


\section{Birkin et al.}

that has undergone no special treatment (such as degassing, deionising) under atmospheric pressure. For such conditions the threshold acoustic pressure to generate inertial cavitation is around $\sim 100-120 \mathrm{kPa}$ (zero-to-peak amplitude), and as the driving pressure exceeds this, a relatively broad range of initial bubble sizes (from microns to tens of microns radius) will generate inertial cavitation ${ }^{9}$. However, this familiar scheme of single bubble dynamics has only limited applicability to a complex, though common, cavitation environment such as the horn produces, and attention must also be paid to the interactions between bubbles, and the roles of the bubbles whose size and location place them below the threshold for inertial cavitation. The distinction between inertial and non-inertial events is vital when interpreting results of sonochemical/sonoelectrochemical experiments, as many of the phenomenological effects associated with sonication occur during inertial bubble collapses ${ }^{10}$. Examples inertial cavitation effects include local hot spots ${ }^{11-13}$, light emission ${ }^{14-16}$, radical generation ${ }^{17-19}$, shock waves ${ }^{20,21}$ and material erosion ${ }^{22-24}$ or production ${ }^{25}$. These effects are potentially useful within a chemical environment where, for example, the processing ${ }^{26}$ of materials or the destruction of organic pollutants is desired ${ }^{27-29}$. However, non-inertial cavitation can affect inertial cavitation in many ways, for example by shielding the sound field, setting up microstreaming currents which affect the transport of bubble nuclei and dissolved gases etc ${ }^{30,31}$. In addition solution constituents, for example surfactants, have been shown to influence the phenomena observed ${ }^{32,33}$.

In order to assess the activity in such multibubble cavitation fields, because of the pressure dependence in the fate of cavitation bubbles, the first consideration should be to the shape and characteristics of the sound field generated by the sound source ${ }^{1}$. For an ultrasonic horn, the direct field falls dramatically with distance, such that with increasing ranges its contribution compared to the reverberant and scattered fields reduces. Normally therefore, as the distance from the surface of the sound source is increased, there will be a point at which inertial cavitation 


\section{Birkin et al.}

ceases to exist and only non-inertial bubble oscillation occurs. However, this simple model is complicated by the interacting dynamics of bubbles in clouds ${ }^{34}$ or clusters ${ }^{23,35,36}$, and the fact that the pressure (and flow) fields that drive those bubble dynamics can contain important contributions in addition to the emitted field which would be modelled from the horn, or measured from the horn in bubble-free conditions. Figure 1 demonstrates an example where higher frequency bubble-generated acoustic waves are important in demarcating the extent of the zone close to the horn where inertial cavitation can occur ${ }^{9,21}$. Figure 1 (a)-(e) shows how the high frequency components are effectively scattered off of the electrodes support and contribute significantly to the MBSL spatial distribution of the system (see Fig. 1 (e) for example). The presence of such higher-frequency components in the pressure field, is clear in Fig. 1(f) when a hydrophone was placed within the sound field. In addition, when the horn was operated in identical conditions except that the water used in Fig. 1(f) was replaced in (g) by castor oil, the measured pressure field in this case resembled the sinusoidal voltage signal supplied to the horn but in the absence of the pressure spikes caused by cavitation. The overall cavitational activity is therefore dependent on the cavitation cloud dynamics, and on components in the pressure field other than the direct radiation from the source ${ }^{21,37}$. Furthermore, changes in the drive conditions (e.g. amplitude) can change the effects of the cloud (e.g. in scattering, shield, absorbing or enhancing the sound field), such that the observables (such as the location of luminescence) can in turn change $\mathrm{e}^{15,38}$.

Cavitation is complex enough to generate many effects and to cause difficulties when attempts are made to quantify it. Quantification of cavitation is therefore actually the quantification of the effects of cavitation. In addition, this quantification process is most safely done by the monitoring of numerous effects and comparing the results. Some common methods of quantification of the effects of cavitation (such as sonochemical yield or erosive mass loss) 
Birkin et al.

afford poor spatial and temporal resolution. Measurements which afford fine spatial resolution in conjunction with the correlation of time resolved measures of cavitation ${ }^{39-41}$ provide particularly useful approach to probing such complex cavitation environments, particularly when those measurements cane be made simultaneously.

This manuscript reports the results from a unique combination of time resolved techniques, which are used to characterize the dynamics of cavitation induced close to the tip of an ultrasonic horn which generated the features in Fig. 1. The techniques used are: electrochemical detection of surface erosion, time resolved MBSL, laser scattering, acoustic pressure measurements and highspeed imaging. By combining these techniques it has been possible to gain an insight in to the dynamics of the bubble population and partially elucidate mechanisms associated with the phenomenological effects associated with inertial cavitation. 
Birkin et al.

\section{Experimental}

\section{A. The driving conditions}

A commercial ultrasonic horn (Adaptive Biosystems) fitted with a $3 \mathrm{~mm}$ diameter titanium tip was driven by a continuous sinusoidal signal from a function generator (Grundig Digimess FG 100) via a power amplifier (Brüel \& Kjær Type 2713). The function generator was interfaced with a PC using software written in-house, allowing the frequency, power and duration of the ultrasound to be controlled. The nominal frequency and reference intensity (measured using a

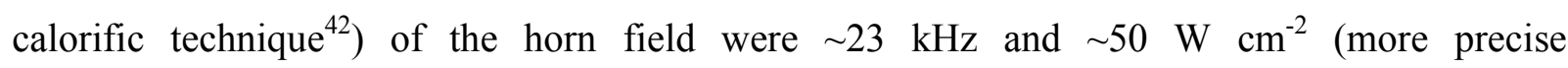
measurements are included in each relevant figure caption).

\section{B. Acoustoelectrochemical Experiments}

Acoustoelectrochemistry is the process by which mass flux ${ }^{43-45}$, reaction ${ }^{46,47}$ or erosion ${ }^{48}$ resulting from acoustically-induced liquid motion (usually bubble-generated motion ${ }^{49}$ ) is monitored by their effects on an electrochemical current, as sensed at an electrode. The detection of mass flux is sensed because, in stagnant fluid, the electrochemical current is limited by diffusion alone, and over time the region of liquid close to the electrode tip becomes depleted of active species. Liquid motion disrupts this depletion layer by introducing convection to the system ${ }^{49,50}$. This is sensed as an enhancement of electrochemical current. The working electrode (or sensing element) is usually made of some noble metal (such as platinum) imbedded in an insulator (such as glass or epoxy resin). In contrast, the electrodes used to sense erosion are made of a passive metal (such a lead in sulphate ${ }^{9,48}$ media or aluminium ${ }^{51}$ ). Erosion is sensed through the current required to regrow a passivating layer which has been mechanically removed from the surface of the electrode. These sensors can have resolutions better than $100 \mu \mathrm{m}$ and $100 \mu \mathrm{s}$ (depending on the mechanism 


\section{Birkin et al.}

investigated). In this manuscript the investigation of the erosion technique is reported in relation to the temporal dynamics of the events generated. Figure 2 shows a schematic representation of the electrochemical rig employed. The working electrode was inserted in the bottom of the electrochemical cell, which itself was placed on an XY stage (Photon Control). The stage allowed $25 \mathrm{~mm}$ of movement in each direction with $0.01 \mathrm{~mm}$ resolution, enabling the position of the working electrode to be accurately controlled in a plane below the tip of the ultrasonic horn. The position of the horn was fixed in the XY plane but controlled in the $\mathrm{Z}$ direction by means of a micrometer and stage (Newport), which allowed $25 \mathrm{~mm}$ of movement with $0.02 \mathrm{~mm}$ resolution. This allowed the separation between the surface of the working electrode and the tip of the ultrasonic horn to be controlled with the necessary high degree of precision.

\section{Acoustic Measurements}

All acoustic pressures were measured with a Brüel \& Kjær type 8103 hydrophone and Brüel \& Kjær type 2635 charge amplifier. The data was recorded by means of an oscilloscope (Le Croy 9310 AM or Tektronix TDS 220). Whilst the type 8103 is a relatively small hydrophone, the active element (a cylinder measuring $6 \mathrm{~mm} \times 6 \mathrm{~mm}$ ) ${ }^{52}$ is still large compared to the pressure gradients expected. Consideration of this in terms of spatial averaging effects is paramount when recording and interpreting pressure measurements. However, the element is small enough to satisfy the condition that the dimensions are less than one tenth of the wavelength of the driving sound wave used in this work $(\sim 7 \mathrm{~cm})$, which implies that the hydrophone is omnidirectional with respect to the driving $(\sim 23 \mathrm{kHz})$ pressure wave. However, for frequencies above $180 \mathrm{kHz}$ these hydrophones are known to be limited, with loss in sensitivity, and their phase response is 
Birkin et al.

rarely calibrated. Hence, measurements of shock pressure amplitudes will be underestimated under these conditions.

\section{Luminescence Experiments}

The temporal characteristics of MBSL were investigated using an EMI-Gencon Inc. RFI/B-293 photomultiplier tube (PMT) powered by $2 \mathrm{kV}$ power supply (Brandenburg 475R). The PMT was fixed to the underside of a wooden platform, which had a hole $(10.5 \mathrm{~cm}$ diameter $)$ in the centre. A sliding cover allowed the hole to be covered when not in use. The cell, which was a Pyrex crystallisation dish (internal diameter, $93 \mathrm{~mm}$ depth, $50 \mathrm{~mm}$ ), was placed above the PMT and the horn was positioned such that the tip was $15 \mathrm{~mm}$ below the surface of the solution $\left(0.75 \mathrm{~mol} \mathrm{dm}^{-}\right.$ ${ }^{3} \mathrm{Na}_{2} \mathrm{SO}_{4}$ ) and directly above the PMT. In order to measure the pressure and light output simultaneously, a hydrophone was placed in the cell such that the acoustic centre was at a lateral distance of $15 \mathrm{~mm}$ from the centre of the tip of the horn. An oscilloscope (Tektronix TDS 224) was used to record the output from the PMT and the hydrophone simultaneously, allowing the pressure signal and light output to be temporally correlated.

\section{E. High-Speed Imaging}

High-speed video footage was recorded using a Photosonics Phantom V7 or Photron APX RS (both capable of frame rates exceeding 100000 frames per second (fps)) digital video camera fitted with a monozoom lens. It was necessary to backlight the subject so that the horn and bubbles appear as silhouettes. For high-speed imaging experiments a cylindrical glass cell (75 $\mathrm{mm}$ i.d, $105 \mathrm{~mm}$ height) fitted with a flat window was used.

\section{F. Laser Scattering Experiments}




\section{Birkin et al.}

For laser scattering experiments a $3 \mathrm{dm}^{3}$ beaker was modified by the scientific glass blowing service in the School of Chemistry at the University of Southampton to increase the height and volume to $3.5 \mathrm{dm}^{3}$, and include optically flat windows $(4 \mathrm{~cm}$ diameter) on opposite sides of the beaker. Also included was a hollow glass support, which was terminated with an SQ 13 fitting, allowing a hydrophone to be positioned in the centre of the beaker at a height of $12 \mathrm{~cm}$ from the base. The overall height of the beaker was $23 \mathrm{~cm}$ and the internal radius was $7 \mathrm{~cm}$. The beaker was filled to a height of $19 \mathrm{~cm}$ with pure water and the ultrasonic horn was positioned such that it was in the centre of the beaker submerged to a depth of $15 \mathrm{~mm}$. A laser (UG 5001-21 diode laser, $1 \mathrm{~mW}, 635 \mathrm{~nm}, \mathrm{RS}$ ) was shone through the beaker and detected by means of a photodiode (AEPX65, RS) and an amplifier circuit made in-house ${ }^{53}$. The photodiode had an active area of $0.8 \mathrm{~mm}^{2}$. The electronics were such that the maximum output (under direct illumination by the laser) was $-1.2 \mathrm{~V}$. Any scattering of the beam resulted in an increase in the output voltage (i.e. the voltage became less negative). Prior to each experiment, the position of the photodiode was adjusted by means of an XYZ stage (Newport, $0.02 \mathrm{~mm}$ resolution) such that the output was at a maximum negative value. The laser beam was flat with a width of $3 \mathrm{~mm}$ and was orientated such that it was parallel with the face of the ultrasonic horn. The distance between the horn and the beam was determined by lowering the horn (by means of a micrometer and stage) until it just blocked the beam and then retracting the horn a known distance. The actual distance used is given in the appropriate figure legends. The pressure and photodiode output were recorded using a Le Croy 9310 AM oscilloscope. For experiments where the distance between the horn and the hydrophone was varied, the glass support was blanked off and the hydrophone was clamped in place using a retort stand.

\section{G. Chemicals and Solutions}


Birkin et al.

All solutions were made up using water from an USF Elga Purelab Option E10 water purification system. Water purified in this manner had a conductivity of below $0.1 \mu \mathrm{S} \mathrm{cm}^{-1}$ and a low organic content (manufacture quoted TOC $<30 \mathrm{ppb}) . \mathrm{Na}_{2} \mathrm{SO}_{4}(\mathrm{BDH}$, AnalaR) was used as received. 
Birkin et al.

\section{Results and Discussion}

\section{A. High Speed Imaging and Laser Scattering}

In order to study the dynamics of the bubble population, first high-speed imaging was employed. Figure 3 shows a set of images recorded using a high-speed camera of the region below the operating ultrasonic horn. Here the tip of the sound source is seen in the upper centre of each image. The framing rate (100000 f.p.s) is high enough to resolve the growth and collapse of a cluster of bubbles located on the tip of the sound source. Interestingly this bubble cloud appears to grow and collapse periodically (with a period close to $130 \mu \mathrm{s}$ ). However, the period of this motion does not match the period of the oscillation of the ultrasonic probe's tip (in this case $\sim 43$ $\mu$ s or a frequency of $\sim 23 \mathrm{kHz}$ ). Rather, Fig. 3 shows that the collapse process occurs over $\sim 130$ $\mu \mathrm{s}$ or three sound cycle periods. This can be seen as the 13 frame interlude between complete clearing of the cluster of bubbles from the tip (see highlighted frames 7, 20 and 33). The repetitive nature of these events at $1 / 3$ the fundamental frequency, match the periodic shock wave like emission in the acoustic output of the system, which has been reported previously (see fig. 1 and $\mathrm{ref}^{9}$ ). Note that in the absence of cavitation (e.g. by suppressing this process using castor oil instead of water - see Fig. $1(\mathrm{~g}))$ the pressure wave became sinusoidal in nature ${ }^{9}$. This indicates that the shock wave like emission is strongly linked to the cavitation process within the water rather than an intrinsic property of the transducer.

The periodic nature of the bubble events recorded using this high-speed camera technique was investigated further using a laser scattering technique. Here the beam of a diode laser was passed through the solution at a defined distance below the tip of the operating ultrasonic horn. A photo diode was then used to detect the intensity of the beam. In the absence of any scattering objects 


\section{Birkin et al.}

in the laser beam's path (e.g. bubbles) the voltage signal recorded from the photodiode is at its most negative. However, scattering due to bubbles or other objects is seen as a reduction in the negative voltage (towards zero when the laser beam is totally obscured) recorded by the photo diode. Figure 4 shows a typical signal obtained from the photodiode as a function of time during operation of the ultrasonic horn. In this experiment a hydrophone was also placed in the cell to record simultaneously the acoustic pressure amplitude as a function of time. Initially the value of $V_{P D}$ is $-0.7 \mathrm{~V}$. This indicates that there is some scattering of the laser beam. The value of $V_{\mathrm{PD}}$ is then seen to decrease, reaching a minimum value of approximately $-1.2 \mathrm{~V}$ corresponding to clear passage of the laser beam through the solution below the tip of the probe. This is ascribed to the absence of a cluster of cavitation bubbles, such as the situation shown in Fig. 3 frames 7, 20 and 33. Interestingly, this solution clearing is almost immediately followed (i.e. within $10 \mu \mathrm{s}$ ) by a sharp rise or spike in the photodiode signal (labelled A). The output then increases to the original value (labelled C) before this cycle of decrease, spike $\left(A^{\prime}\right)$ and increase is repeated. The period of these events is $\sim 170 \mu$ s (or 4 cycles of the ultrasonic wave employed). The events (labelled A and $A^{\prime}$ ) correspond to the situation when the laser was blocked by an object, presumably a bubble or bubble cloud. At the same time the hydrophone data gives an insight into the origin of the effects observed with the laser scattering experiment. About $19 \mu$ s after each event of periodic clearing and sudden spike in the laser signal (labelled $A$ and $A^{\prime}$ ) there is a spike in the pressure signal (labelled B and $B^{\prime}$ ). The clarity of the shock emission is obscured by the reverberant nature of the relatively small vessel $\left(\sim 3.5 \mathrm{dm}^{3}\right)$ employed in the experiments. However, clearer evidence for this pressure wave (the exact profile of which is probably not captured by the hydrophone because of the latter's amplitude and phase response at high frequencies) has been reported previously in a larger vessel $\left(\sim 2 \mathrm{~m}^{3}\right)$ with the hydrophone placed closer to the tip of the 


\section{Birkin et al.}

sound source (see Fig. 1 and ref ${ }^{9}$ ). The link between these events and the hydrophone data can be further highlighted by varying the distance between the tip of the ultrasonic probe and the hydrophone and then measuring the delay between the events in the laser scattering experiment and the hydrophone data. The results of such an experiment are shown in Fig. 5 which was performed in the $3.5 \mathrm{dm}^{3}$ vessel. Here the time between the pressure pulse (B) and the spikes in the scattering signal $(A)$ can be seen to increase as the distance between the hydrophone and the tip of the ultrasonic probe was increased. The relationship is linear and can be used to calculate the speed of sound in the liquid as $670 \pm 100 \mathrm{~m} \mathrm{~s}^{-1}$. This is much less than the speed of sound in bulk water but this may be expected in the presence of bubbles. This retardation of sound velocity in bubbly liquids is well known, ${ }^{54}$ and similar values have been measured in vessels in which inertial cavitation has been generated ${ }^{1}$.

While the high-speed imaging shown in Fig. 3 can capture the periodic collapse of a large bubble cloud at the tip of the ultrasonic horn, there is nothing visible to explain the transient laser scattering observed (the spikes labelled $A$ and $A^{\prime}$ in Fig. 4). However, further high-speed imaging experiments have revealed evidence of transient bubble clouds. Figure 6 shows a sequence of frames collected at 100000 fps below an operating ultrasonic horn and above an electrode. The collapse of the cluster formed below the ultrasonic tip can be seen between frames 1 and 7. Frame 8 is almost totally clear. This is consistent with the data presented thus far. However, frame 9 shows a group of small bubbles in the bulk solution (highlighted by the dotted oval). They are also much smaller than the large cluster that exists at the tip of the horn. Such bubbles have been detected in other scenarios ${ }^{40}$, in response to a search for the cause of spikes in sonoluminescence emission $^{41}$. It is proposed that these events are partially responsible for the spike in the laser scattering data (note the shock itself may give a perturbation of the laser through Schlieren effects ${ }^{55}$ ). A further example of these transient clouds of bubbles is shown in 


\section{Birkin et al.}

Fig. 7. This figure shows a set of images, which depict key stages of the bubble population dynamics observed and described above imaged by illumination through the cell to the camera (creating a shadow effect which is useful in imagining bubble produced within the media). Figure 7 (a) shows the cluster at the tip of the horn (labelled BC) and a thinner cloud of bubbles (labelled BN) below the cluster. Figure 7 (a)-(c) shows the bubble cluster collapsing. In Fig. 7 (d) both the bubble cluster on the tip of the horn and the bubble cloud (BN) are no longer visible in the image. Figure 7 (e) shows a transient bubble cloud highlighted in a dotted oval. Both the bubble cluster and the thinner bubble cloud are reformed after the transient bubble cloud event (see Fig. $7(f)-(h)$ ). This agrees with the laser scattering data shown in Fig. 4. However, the images also clearly show that the pre-existing bubbles (see Fig. 7 frame (a), BN) are compressed before a 'rebound' event was observed (see Fig. 7 frame (d)).

\section{B, Correlation of Bubble Dynamics with Physical Effects}

The correlation of these dynamics with the observed effects of cavitation was investigated by first measuring the response of a surface erosion sensitive electrode ${ }^{48,56}$ (in this case a passivated $\mathrm{Pb}$ electrode) within this environment. Figure 8 shows the current time transient recorded for such an electrode in conjunction with the acoustic pressure signal recorded simultaneously with a hydrophone. Note that the lower trace shows the acoustic pressure trace, which is somewhat distorted because this data was recorded in a relatively small cylindrical cell (volume $71.5 \mathrm{~cm}^{3}$ ). As a result the hydrophone was subject to reverberant field effects in addition to the direct field and signals associated with cavitation activity. Nevertheless, pressure spikes (labelled*) can be seen to occur periodically, in a similar fashion to those shown previously. The upper trace shows the current recorded at the same time. The electrode was positioned $1 \mathrm{~mm}$ from the tip of the horn and the hydrophone was $10 \pm 1 \mathrm{~mm}$ from the tip of the horn. In order to correlate the 


\section{Birkin et al.}

current and pressure data $15 \mu$ s has been added to the current time trace. This is to account for the delay caused by the finite speed of sound in the liquid. However, owing to uncertainties in the speed of sound (as a result of the unknown bubble population) and the location of the hydrophone, there is an error of at least $\pm 4 \mu \mathrm{s}$. Nevertheless, a repassivation current time transient can be seen to occur at time $t \sim 210 \mu \mathrm{s}$, which correlates temporally with a pressure spike. A second, much smaller transient can be seen at time $t \sim 380 \mu$ s (4 pressure cycles later), which also correlates with a large pressure spike. While it must be noted that the timing of the pressure spike is by no means exact, it is clear that the erosion of the electrode appears to be associated with the shock wave event. In addition to this erosion data, temporally correlated pressure and scattering, MBSL data was also recorded. Figure 9 shows the MBSL output recorded by means of a photomultiplier tube and the corresponding acoustic pressure time history. Again, light output (negative voltage spikes) correlates temporally with the high pressure spikes and occurs at $1 / 3$ of the fundamental frequency of the horn. This suggests that MBSL is also associated with the events that occur at the time of the subharmonic pressure spike emission. It is interesting to note that, in the case of MBSL, for every subharmonic pressure spike seen in the hydrophone signal there is corresponding light output. In contrast, not every shock wave seen in Fig. 8 leads to a repassivation transient. This suggests that although a global shock wave is generated, which can be detected by the hydrophone, it is the action of a localised event (possible microjetting) that leads to surface erosion. The lead microelectrode represents a small target, whereas the PMT collects light from a wide area. Hence only events, which occur in a suitable location, will be detected by the passivated electrode.

\section{The Mechanism}




\section{Birkin et al.}

The evidence presented thus far suggests that the environment below an operating ultrasonic horn is very complex indeed. Clearly, shock waves, bursts of bubble events, erosion and MBSL emission all occur in a local space and within a short time window. It is interesting to discuss the possible mechanisms responsible for these physical observations. The high-speed imaging shows that a cluster collapse $\mathrm{e}^{23,35,36}$ is produced on the surface of the operating ultrasonic horn. This cluster collapse correlates with the extended periodicity of the other experimental observations (e.g. in this case the erosion, shock and MBSL measurements occur on every $3^{\text {rd }}$ or $4^{\text {th }}$ cycle of the ultrasonic wave in time with the laser scattering and high-speed imaging of the cluster collapse). However, the actual mechanisms responsible for surface erosion and MBSL are less clear. The laser scattering and high-speed imaging suggests that after the large gas cavity on the surface of the horn has collapsed, a cloud of small transient bubbles are observed. These are at an extended distance compared to the cavity collapse itself, which is estimated to extend $\sim 1400$ $\mu \mathrm{m}$ from the surface of the horn. As erosion and MBSL have been observed at greater distances that this ${ }^{9,21}$ one could suggest that these small, transient bubble events are responsible for both effects, or that the transient bubble cloud marks the passage of a pressure wave through the liquid, and the arrival of that pressure wave on, say, the erosion sensor generates further cavitation and thence erosion. It is useful at this point to discuss the sequence of events that could be responsible for these experimental observations.

Inertial collapse of an individual bubble can generate a positive pressure pulse which, close to the bubble, can have amplitude very much greater than that of the wave which caused the collapse ${ }^{57}$, although the amplitude of this wave will of course decay as it propagates to the far field ${ }^{37}$. However, high amplitude tensile components can also be generated, for example as these compressive waves reflect off neighbouring bubbles ${ }^{37,57}$. Furthermore, there will be edge waves 


\section{Birkin et al.}

from the perimeter of the horn face, and a complex of waves generated within (and at the perimeter of) the horn material itself ${ }^{10}$. Hence the collapse of a cluster against the horn face, and interaction of the pressure fields within these media, will generate a complex pressure wave which propagates outwards.

The short lived transparency of the liquid (see Fig. 7 frame (d)) is assumed to coincide with the low laser scattering (see Fig. 4 labelled L) and is a result of compression of bubbles by the above mentioned compressive pulse. However, the limited hydrophone response cannot capture its true form nor that of the subsequent large tension (although both have been observed in the experiments, see Fig. $4 \mathrm{~B}$ and $\mathrm{T}$ for example). Nevertheless, the action of the tension on previously compressed bubbles would be expected to produce the extreme bubble growth and subsequent high transient laser scattering observed. This conclusion is supported ${ }^{58}$ by cavitation histories associated with shock waves, particularly during lithotripsy ${ }^{20,59,60}$.

It is worth noting that only surface erosion and MBSL have been investigated here. However, the consequences for chemical activity within this environment are unclear. If chemical activity is linked to MBSL output of a system, as suggested in other reports ${ }^{18,61,62}$, one would also expect this to follow this cluster collapse and transient bubble cloud mechanism and associated timing. Also, the discussion thus far has concentrated on the space close to the tip of the sound source where inertial events are generated $(<3 \mathrm{~mm}$ away from the centre of the piston like emitter employed here). While this region is rich in mechanistic detail, it only represents a small fraction of the total volume of the liquid. In other locations, outside of this zone, the behaviour of bubbles will be non-inertial but still of interest.

\section{Conclusions}


Birkin et al.

A multi sensor/imaging approach to the environment below an operating ultrasonic horn has been reported. The environment has been shown to be extremely complex with shock waves, inertial cavitation, cluster collapses and transient bubble clouds observed. These observations indicate that physical measurements such as surface erosion and MBSL emission from such an environment is strongly periodic in nature and occur at a subharmonic resonance related to the dynamics of bubble clouds within this media.

\section{Acknowledgements}

We thank the EPSRC for funding (EP/E024408/1 and EP/D05849X/1) and the EPSRC Instrument Loan Pool for assistance with some of the high-speed imaging reported in this manuscript. 
Birkin et al.

\section{References}

${ }^{1}$ P. R. Birkin; T. G. Leighton; J. F. Power; M. D. Simpson; A. M. L. Vinçotte; P. F. Joseph, "Experimental and Theoretical Characterisation of Sonochemical Cells. Part 1. Cylindrical Reactors and Their Use to Calculate the Speed of Sound in Aqueous Solutions.", Journal of Physical Chemistry A. 107, 306-320, (2003).

${ }^{2}$ T. G. Leighton; P. R. White; C. L. Morfey; J. W. L. Clarke; G. J. Heald; H. A. Dumbrell; K. R. Holland, "The effect of reverberation on the damping of bubbles", Journal Of The Acoustical Society Of America. 112, 1366-1376, (2002).

${ }^{3}$ C. K. Holland; R. E. Apfel, "An Improved theory For the Prediction of Microcavitation Thresholds", IEEE Transactions Ultrasonics Ferroelectrics and Frequency Control. 36, 204-208, (1989).

${ }^{4}$ R. E. Apfel; C. K. Holland, "Gauging the Likelihood of Cavitation from Short-Pulse, Low-Duty Cycle Diagnostic Ultrasound", Ultrasound in Medicine and Biology. 17, 179-185, (1991).

${ }^{5}$ H. G. Flynn, "Cavitation Dynamics II. Free pulsations and models for cavitation bubbles", Journal of the Acoustics Society of America. 58, 1160-1170, (1975).

${ }^{6}$ H. G. Flynn, "Cavitation Dynamics I. A mathematical formulation", Journal of the Acoustics Society of America. 57, 1379-1396, (1975).

${ }^{7}$ T. G. Leighton "The Acoustic Bubble, section 4.3.1"; (Academic Press, London, 1994); 312-329.

${ }^{8}$ T. G. Leighton, "Bubble Population Phenomena in Acoustic Cavitation", Ultrasonics Sonochemistry. 2, S123-S136, (1995).

${ }^{9}$ P. R. Birkin; D. G. Offin; T. G. Leighton, "Experimental and theoretical characterisation of sonochemical cells. Part 2: cell disruptors (Ultrasonic horns) and cavity cluster collapse", Physical Chemistry Chemical Physics. 7, 530-537, (2005).

${ }^{10}$ T. G. Leighton, "What is ultrasound?", Progress in Biophysics \& Molecular Biology. 93, 3-83, (2007).

${ }^{11}$ K. S. Suslick, "The Chemical Effects of Ultrasound", Scientific American. 260, 80-86, (1989).

${ }^{12}$ K. S. Suslick, "Sonochemistry", Science. 247, 1439-1445, (1990).

${ }^{13}$ K. S. Suslick; D. A. Hammerton; R. E. Cline, "The Sonochemical Hotspot", Journal of the American Chemical Society. 108, 5641-5642, (1986). 
Birkin et al.

${ }^{14}$ L. A. Crum; G. T. Reynolds, "Sonoluminescence produced by "stable" cavitation", Journal of the Acoustics Society of America. 78, 137-139, (1985).

${ }^{15}$ A. J. Walton; G. T. Reynolds, "Sonoluminescence", Advances in Physics. 33, 595-660, (1984).

${ }^{16}$ S. Putterman; P. G. Evans; G. Vazquez; K. Weninger, "Cavitation science - Is there a simple theory of sonoluminescence?", Nature. 409, 782-783, (2001).

${ }^{17}$ A. Weissler; H. W. Cooper; S. Snyder, "Chemical effect of ultrasonic waves: oxidation of potassium iodide solution by carbon tetrachloride", Journal of the American Chemical Society. 72, 1769-1775, (1950).

${ }^{18}$ P. R. Birkin; J. F. Power; A. M. L. Vinçotte; T. G. Leighton, " A 1 kHz frequency resolution study of a variety of Sonochemical Processes", Physical Chemistry Chemical Physics. 5, 4170-4174, (2003).

${ }^{19}$ G. Mark; A. Tauber; R.Laupert; H. P. Schuchmann; D. Schulz; A. Mues; C. v. Sonntag, "OH-radical formation by ultrasound in aqueous solution - Part II:Terephthalate and Fricke dosimetry and the influence of various conditions on sonolytic yield", Ultrasonics Sonochemistry. 5, 41-52, (1998).

${ }^{20}$ B. Wolfrum; T. Kurz; R. Mettin; W. Lauterborn, "Shock wave induced interaction of microbubbles and boundaries", Physics of Fluids. 15, 2916-2922, (2003).

${ }^{21}$ P. R. Birkin; D. G. Offin; P. F. Joseph; T. G. Leighton, "Cavitation, shock waves and the invasive nature of sonoelectrochemistry", Journal of Physical Chemistry B. 109, 16997-17005, (2005).

${ }^{22}$ F. J. Heymann "Towards Quantitative Prediction of Liquid impact Erosion"; (ASTM Special Technical Publication, Philadelphia, 1969); 212-248.

${ }^{23}$ I. Hansson; K. A. Morch, "The dynamics of cavity clusters in ultrasonic (vibratory) cavitation erosion", Journal of Applied Physics. 51, 4651-4658, (1980).

${ }^{24}$ R. Garcia; F. G. Hammitt; R. E. Nystrom "Correlation of cavitation Damage with Other Material and Fluid Properties"; (ASTM Special Technical Publication, Philadelphia, 1967); 239-279.

${ }^{25}$ K. Okitsu; M. Ashokkumar; F. Grieser, "Sonochemical synthesis of gold nanoparticles: Effects of ultrasound frequency", Journal of Physical Chemistry B. 109, 20673-20675, (2005).

${ }^{26}$ Y. G. Adewuyi, "Sonochemistry: Environmental science and engineering applications", Industrial \& Engineering Chemistry Research. 40, 4681-4715, (2001). 


\section{Birkin et al.}

${ }^{27}$ J. Berlan; F. Trabelsi; H. Delmas; A. M. Wilhelm; J. F. Petrignani, "Oxidative degradation of phenol in aqueous media using ultrasound", Ultrasonics Sonochemistry. 1, s97-s102, (1994).

${ }^{28}$ M. R. Hoffmann; I. Hua; R. Hoechemer, "Application of ultrasonic irradiation for the degradation of chemical contaminants in water", Ultrasonics sonochemistry. 3, s163-s172, (1996).

${ }^{29}$ M. E. Abdelsalam; P. R. Birkin, "A study investigating the sonoelectrochemical degradation of an organic compound employing Fenton's reagent.", Physical Chemistry Chemical Physics. 4, 5340-5345, (2002).

${ }^{30}$ P. R. Birkin; T. G. Leighton; Y. E. Watson, "The use of acoustoelectrochemistry to investigate rectified diffusion", Ultrasonics Sonochemistry. 11, 217-221, (2004).

${ }^{31}$ M. J. W. Pickworth; P. P. Dendy; T. G. Leighton; A. J. Walton, "Studies of the cavitational effects of clinical ultrasound by sonoluminescence: 2 Thresholds for sonoluminescence from a therapeutic ultrasound beam and the effect of temperature and duty cycle", Physics in Medicine and Biology. 33, 1249-1260, (1988).

${ }^{32}$ M. Ashokkumar; F. Grieser, "The effect of surface active solutes on bubbles in an acoustic field", Physical Chemistry Chemical Physics. 9, 5631-5643, (2007).

${ }^{33}$ A. Brotchie; M. Ashokkumar; F. Grieser, "Effect of water-soluble solutes on sonoluminescence under dualfrequency sonication", Journal of Physical Chemistry C. 111, 3066-3070, (2007).

${ }^{34}$ Z. Zeravcic; D. Lohse; W. van Saarloos, "Collective oscillations in bubble clouds", Journal of Fluid Mechanics. 680, 114-149, (2011).

${ }^{35}$ B. Vyas; C. M. Preece, "Stress produced in a solid by cavitation", Journal of Applied Physics. 47, 5133-5138, (1976).

${ }^{36}$ I. Hansson; V. Kedrinskii; K. A. Morch, "On the dynamics of cavity clusters", Journal of Physics D: Applied Physics. 15, 1725-1734, (1982).

${ }^{37}$ A. R. Jamaluddin; G. J. Ball; C. K. Turangan; T. G. Leighton, "The collapse of single bubbles and calculation of the far-field acoustic emissions for cavitation induced by shock wave lithotripsy", Journal of Fluid Mechanics. 677, 305-341, (2011).

${ }^{38}$ N. C. Eddingsaas; K. S. Suslick, "Evidence for a plasma core during multibubble sonoluminescence in sulfuric acid", Journal of the American Chemical Society. 129, 3838-3839, (2007). 
Birkin et al.

${ }^{39}$ C. J. B. Vian; P. R. Birkin; T. G. Leighton, "Cluster collapse in a cylindrical cell: Correlating multibubble sonoluminescence, acoustic pressure and erosion", Journal of Physical Chemistry C. 114, 16416-16425, (2010).

${ }^{40}$ T. G. Leighton; A. J. Walton; J. E. Field, "High-speed photography of transient excitation", Ultrasonics. 27, 370373, (1989).

${ }^{41}$ T. G. Leighton, "Transient excitation of insonated bubbles", Ultrasonics. 27, 50-53, (1989).

${ }^{42}$ T. J. Mason; J. P. Lorimer; D. M. Bates, "Quantifying Sonochemistry - casting some light on a black art", Ultrasonics. 30, 40-42, (1992).

${ }^{43}$ F. Marken; J. C. Eklund; R. G. Compton, "Voltammetry in the presence of ultrasound", Journal of Electroanalytical Chemistry. 395, 335-339, (1995).

${ }^{44}$ H. H. Zhang; L. A. Coury, "Effects of High-Intensity Ultrasound on Glassy-Carbon Electrodes", Analytical Chemistry. 65, 1552-1558, (1993).

${ }^{45}$ C. R. S. Hagan; L. A. Coury, "Comparison of Hydrodynamic Voltammetry Implemented by Sonication to a Rotating-Disk Electrode", Analytical Chemistry. 66, 399-405, (1994).

${ }^{46}$ P. R. Birkin; J. F. Power; T. G. Leighton, "Electrochemical evidence of H produced by ultrasound", Journal of the Chemical Society Chemical Communications. 2230-2231, (2001).

${ }^{47}$ P. R. Birkin; J. F. Power; T. G. Leighton; A. M. L. Vinçotte, "Cathodic Electrochemical Detection of Sonochemical Radical Products.", Analytical Chemistry. 74, 2584-2590, (2002).

${ }^{48}$ P. R. Birkin; R. O'Connor; C. Rapple; S. Silva-Martinez, "Electrochemical measurement of erosion from individual cavitation generated from continuous ultrasound", Journal of the Chemical Society Faraday Transactions. 94, 33653371, (1998).

${ }^{49}$ Y. E. Watson; P. R. Birkin; T. G. Leighton, "Electrochemical detection of Bubble Oscillation", Ultrasonics Sonochemistry. 10, 65-69, (2003).

${ }^{50}$ P. R. Birkin; S. Silva-Martinez, "The Effect of Ultrasound on Mass Transfer to a Microelectrode.", Journal of the Chemical Society Chemical Communications. 1807 - 1808, (1995).

${ }^{51}$ C. J. B. Vian; P. R. Birkin; T. G. Leighton, "Opto-Isolation of Electrochemical Systems in Cavitation Environments", Analytical Chemistry. 81, 5064-5069, (2009).

52"Application Notes-Introduction to Underwater Acoustics"; (Brüel \& Kjær (Nærum Offset), Nærum, 1-27. 
Birkin et al.

${ }^{53}$ D. G. Offin. "An investigation of fast surface re-formation in the presence of inertial (transient) cavitation". PhD Thesis, University of Southampton, 2006.

${ }^{54}$ F. E. Fox; S. R. Curley; G. S. Larson, "Phase Velocity and Absorption Measurements in Water Containing Air Bubbles ", Journal of the Acoustics Society of America. 27, 537-539, (1955).

${ }^{55}$ J. Noack; A. Vogel, "Single-shot spatially resolved characterization of laser-induced shock waves in water", Applied Optics. 37, 4092-4099, (1998).

${ }^{56}$ P. R. Birkin; D. G. Offin; T. G. Leighton, "A novel dual microelectrode for investigating mass transfer and surface erosion caused by cavitation", Electrochemistry Communications. 6, 1174-1179, (2004).

${ }^{57}$ C. K. Turangan; A. R. Jamaluddin; G. J. Ball; T. G. Leighton, "Free-Lagrange simulations of the expansion and jetting collapse of air bubbles in water", Journal of Fluid Mechanics. 598, 1-25, (2008).

${ }^{58}$ T. G. Leighton; F. Fedele; A. Coleman; C. McCarthy; S. Ryves; A. Hurrell; A. De Stefano; P. R. White, "A passive acoustic device for real-time monitoring the efficacy of shockwave lithotripsy treatment", Ultrasound in Medicine and Biology. 34, 1651-1665, (2008).

${ }^{59}$ R. O. Cleveland; O. A. Sapozhnikov; M. R. Bailey; L. A. Crum, "A dual passive cavitation detector for localized detection of lithotripsy-induced cavitation in vitro", Journal of the Acoustical Society of America. 107, 1745-1758, (2000).

${ }^{60}$ D. S. Campbell; H. G. Flynn; D. T. Blackstock; C. Linke; E. L. Carstensen, "The Acoustic Fields of the Wolf Electrohydraulic Lithotripter", Journal of Lithotripsy \& Stone Disease. 3, 147-156, (1991).

${ }^{61}$ M. A. Beckett; I. Hua, "Impact of ultrasonic frequency on aqueous sonoluminescence and sonochemistry", Journal of Physical Chemistry A. 105, 3796-3802, (2001).

${ }^{62}$ A. M. L. Vinçotte. "A Frequency study of Sonoluminescence and Sonochemical Activity". MPhil, University of Southampton, 1999.

${ }^{63}$ See supplementary material at [URL will be inserted by AIP] for an example of high-speed imaging of the cluster collapse and the transient bubble cloud. 
Birkin et al.

\section{Figure legends}

Figure 1 A $3 \mathrm{~mm}$ diameter ultrasonic horn $(\mathrm{H})$ is photographed in daylight without (panel (a)), then with (panel (c)), an erosion sensor placed under it (the sensor, labelled E, consists of a 25 micron diameter stainless steel vertical wire embedded in a glass tube). Panels (b) and (d) show the light emission (10 min exposures) from cavitation without (panel (b)) and with (panel (d)) the erosion sensor in place, for continuous ultrasonic irradiation $(22.83 \mathrm{kHz}$, nominal faceplate intensity $56 \pm 5 \mathrm{~W} \mathrm{~cm}^{-2}, \sim 30 \mathrm{kPa}$ zero-to-peak pressure amplitude at $4.5 \mathrm{~mm}$ below the horn). Subtraction of panel (c) from panel (d) leaves a light remnant (panel (e)), indicating that scattering of the acoustic field from the electrochemical sensor is increasing the acoustic pressure in the liquid sufficient to cause inertial cavitation at ranges from the sound source where, without such scattering, the amplitude is insufficient to generate inertial cavitation. The scattered fields generating this effect are not the direct field produced by the horn (which has a wavelength much larger than the sensor), but instead the higher frequency components generated by the cavitation generated. Experiment undertaken in $0.75 \mathrm{~mol} \mathrm{dm}^{-3} \mathrm{Na}_{2} \mathrm{SO}_{4}$ at $25{ }^{\circ} \mathrm{C}$ in aerobic solutions ${ }^{21}$. Panel (f) shows the acoustic pressure $(P)$ time history recorded below an ultrasonic horn using a calibrated hydrophone in a water tank above the inertial cavitation threshold. The distance between the tip of the ultrasonic source and the acoustic centre of the hydrophone was $8.8 \mathrm{~mm}$. Panel $(\mathrm{g})$ shows a plot of the acoustic pressure $(P)$ time signal recorded in castor oil in a $5 \mathrm{dm}^{3}$ beaker. The distance between the active element of the hydrophone and the source was $4.8 \mathrm{~mm}$ (see ref ${ }^{9}$ for further details). 


\section{Birkin et al.}

Figure 2 Illustration of the acoustoelectrochemical set-up employed to record the electrochemical response of the working electrode (WE) as a function of position with respect to the sound source. Note CE and RE refer to the counter and reference electrode respectively.

Figure 3 Image showing 40 consecutive frames taken at $100000 \mathrm{fps}$ showing the tip of an ultrasonic horn (dark oblong at the top of each frame) operating at $22.85 \mathrm{kHz}$. The scale bar in frame 1 represents $1.5 \mathrm{~mm}$ and the white dotted outline shows the solid/liquid boundary of the piston like emitter. The dark outlines show frames (see frames 7, 20 and 33) where the solution is apparently clear. The cell contained aerobic aqueous media at $18-23^{\circ} \mathrm{C}$.

Figure 4 Plot showing the acoustic pressure $(--, P)$ and output from a photodiode $\left(-, V_{\mathrm{PD}}\right)$ recorded simultaneously under exposure to ultrasound $(23.10 \mathrm{kHz})$. The photodiode was aligned with a laser beam, which was passing below the tip of the horn at a distance of $1.4 \mathrm{~mm}$. The distance between the horn and the hydrophone was $20 \pm 1 \mathrm{~mm}$. The cell contained aerobic purified water at $18-23^{\circ} \mathrm{C}$.

Figure 5 Plot showing the acoustic pressure $(P,--)$ and output from a photodiode $\left(V_{\mathrm{PD}},-{ }_{-}\right)$ recorded simultaneously under exposure to ultrasound $(22.83 \mathrm{kHz}$, nominal faceplate intensity 56 $\pm 5 \mathrm{~W} \mathrm{~cm}^{-2}, \sim 30 \mathrm{kPa}$ zero-to-peak pressure amplitude at $4.5 \mathrm{~mm}$ below the horn) at three horn-tohydrophone distances (a) $14.2 \mathrm{~mm}$, (b) $28.2 \mathrm{~mm}$ and (c) $52.2 \mathrm{~mm}$. The cell contained aerobic purified water at $18-23^{\circ} \mathrm{C}$.

Figure 69 frames taken from high speed video footage recorded at $100000 \mathrm{fps}$ of the tip of the horn and an electrode during exposure to ultrasound $(22.83 \mathrm{kHz}$, nominal faceplate intensity $56 \pm$ 
Birkin et al.

$5 \mathrm{~W} \mathrm{~cm}^{-2}, \sim 30 \mathrm{kPa}$ zero-to-peak pressure amplitude at $4.5 \mathrm{~mm}$ below the horn). The tip of the horn is at the top of each frame (shown by the dotted line in frame 1). A dual electrode containing a $\mathrm{Pb}(125 \mu \mathrm{m}$ diameter) and a $\mathrm{Pt}$ (50 $\mu \mathrm{m}$ diameter) wires (labelled in frame 1) can be seen in the bottom of each frame. The scale bar in the bottom left corner of frame 1 represents $250 \mu \mathrm{m}$.

Figure 7. Representative images viewed from the side of the cavity collapse sequence and zones of cavitation events. Image (a) shows the large cavity (labelled BC) on the tip of the piston like emitter (border highlighted by dotted line in frame (b)). Image (a) also shows a cloud of smaller unresolved bubbles (labelled BC) below the large cluster. Image (d) shows the disappearance of the cavity cluster $(\mathrm{BC})$ and bubble cloud $(\mathrm{BN})$ in response to the pressure emissions generated. Image (e) shows the transient cloud (highlighted with a dotted oval) formed after the disappearance of the cluster. Images (f)-(h) show the system has returned to the initial state (e.g. a bubble cluster and cloud). Note the scale bar in frame (h) represents $1 \mathrm{~mm}$. The frame rate was $105000 \mathrm{fps}$ with an exposure time of $2 \mu \mathrm{s}$. The cell contained aerobic purified water at $22 \pm 1$ ${ }^{\circ} \mathrm{C}$. Image take using transmission of light through the cell (hence shadow effect observed to highlight bubble cluster) to a Photron APX-RS camera. See supplementary material on the Journal of the Acoustical Society of America (JASA) website ${ }^{63}$ for an example of a movie sequence illustrating the cluster collapse and transient bubble cloud.

Figure 8 Acoustic pressure $(P)$ and current $(i)$ recorded at a passivated lead electrode $(125 \mu \mathrm{m}$ diameter) held at $+0.8 \mathrm{~V}$ vs. SCE in a solution of $0.75 \mathrm{M} \mathrm{Na}_{2} \mathrm{SO}_{4}$ under exposure to ultrasound $\left(23.10 \mathrm{kHz}\right.$, nominal faceplate intensity $\left.56 \pm 5 \mathrm{~W} \mathrm{~cm}^{-2}\right)$. The electrode-to-horn distance was 1 $\mathrm{mm}$ and the horn-to-hydrophone distance was $10 \pm 1 \mathrm{~mm}$. The experiment was performed under 
Birkin et al.

aerobic conditions in a cylindrical cell (45 mm diameter, $45 \mathrm{~mm}$ depth) at $25 \pm 1{ }^{\circ} \mathrm{C}$. Note the $(*)$ refer to the shock waves emitted by the cavity collapse process.

Figure 9 Plot showing the acoustic pressure $\left(P\right.$, upper trace) and output from the PMT ( $V_{\mathrm{PMT}}$, lower trace) as a function of time under exposure to ultrasound $(23.10 \mathrm{kHz}$, nominal faceplate intensity $56 \pm 5 \mathrm{~W} \mathrm{~cm}^{-2}$ ). The horn-to-hydrophone distance was $15 \pm 1 \mathrm{~mm}$. The experiment was performed under aerobic conditions in a cylindrical cell (93 $\mathrm{mm}$ diameter, $50 \mathrm{~mm}$ depth). The cell contained aerobic aqueous $0.75 \mathrm{M} \mathrm{Na}_{2} \mathrm{SO}_{4}$ at $18-23^{\circ} \mathrm{C}$. Note that the $\left({ }^{*}\right)$ refer to the shock waves emitted by the cavity collapse process. 
Birkin et al.
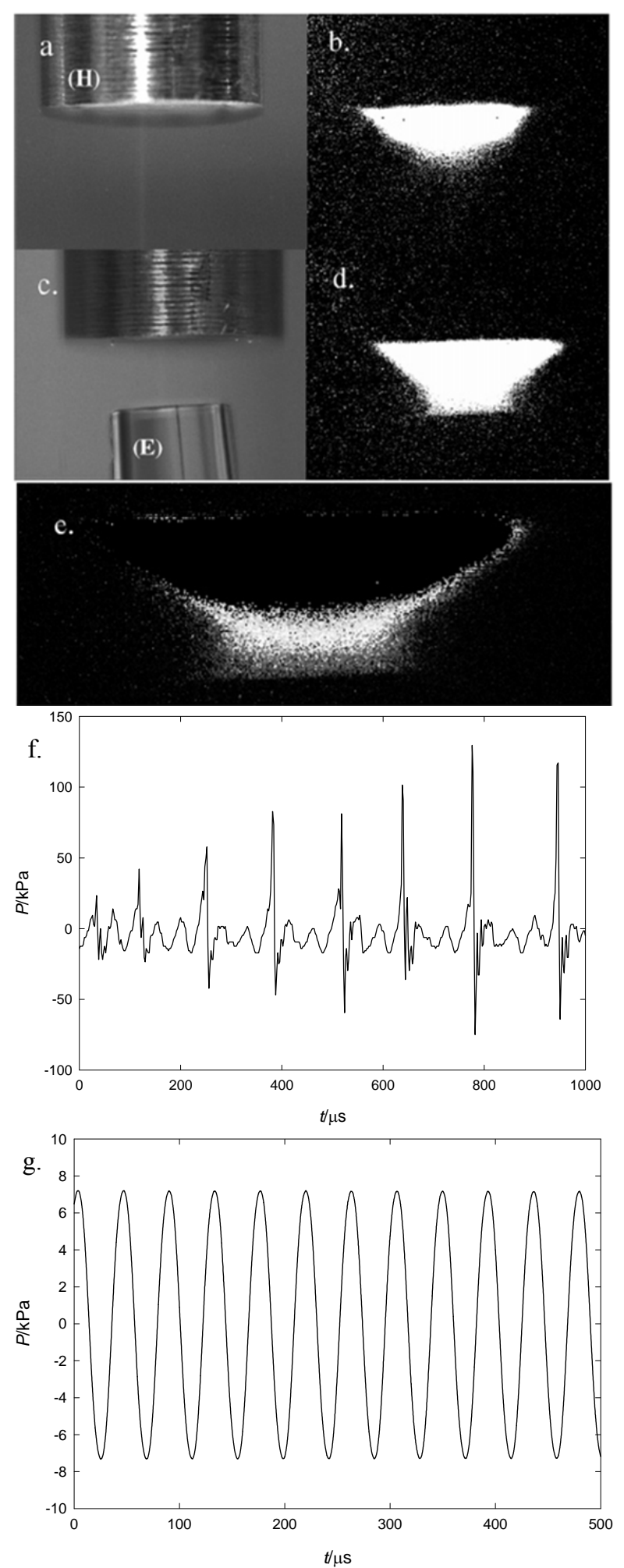

Figure 1 
Birkin et al.

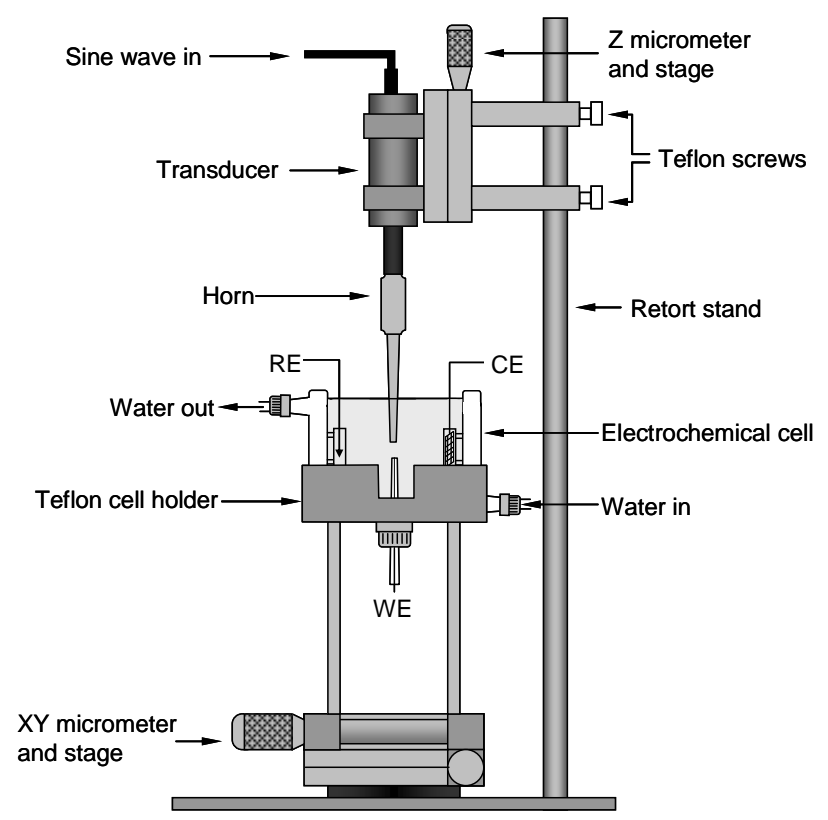

Figure 2 
Birkin et al.

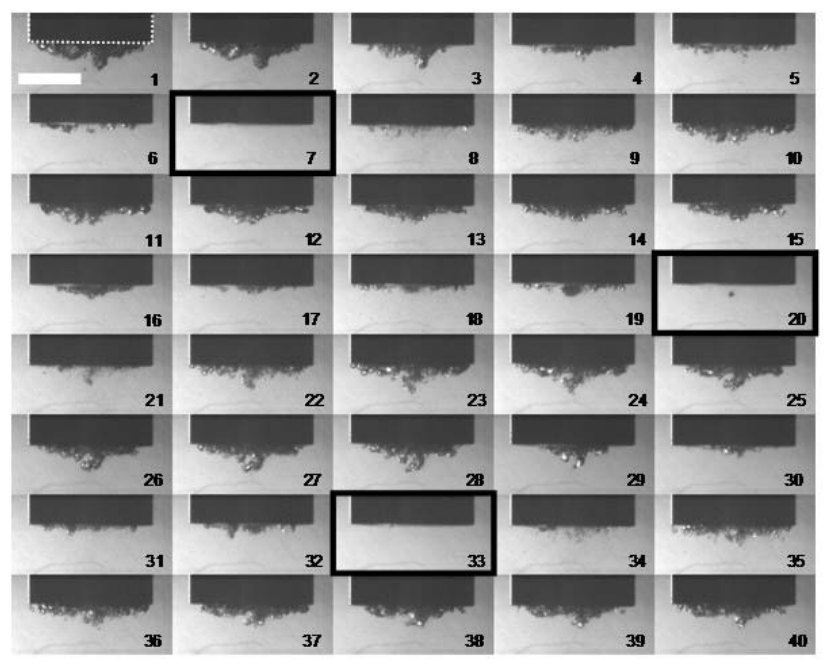

Figure 3 
Birkin et al.

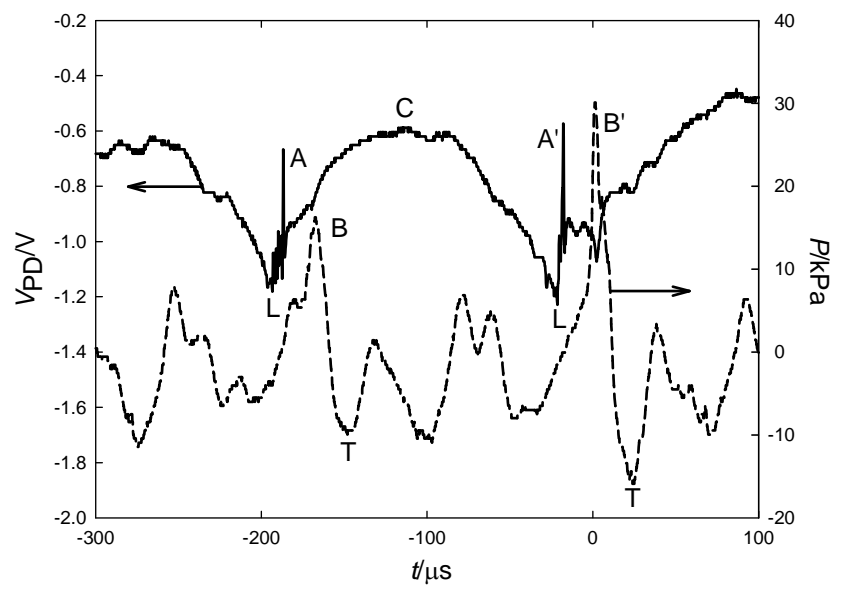

Figure 4 
Birkin et al.
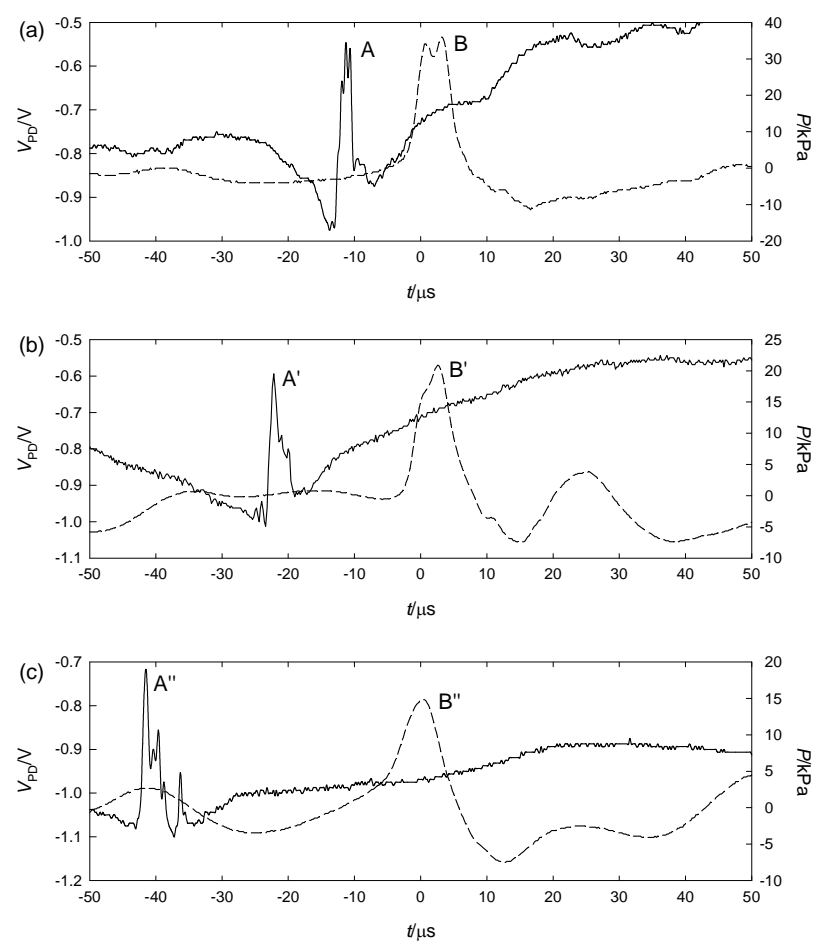

Figure 5 
Birkin et al.

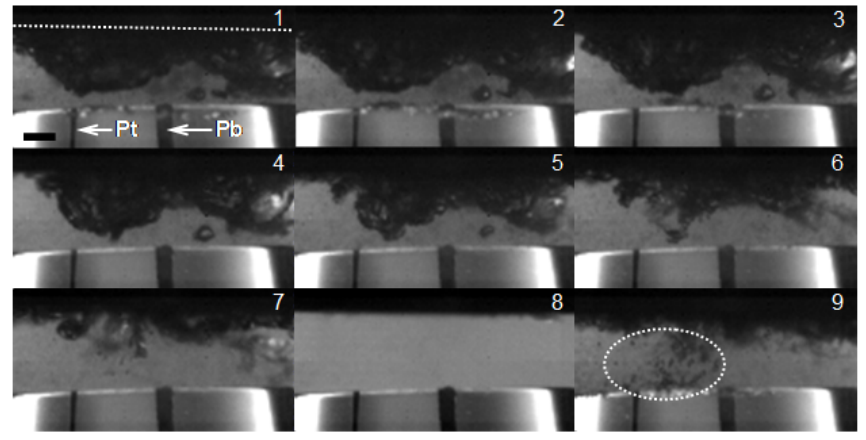

Figure 6 

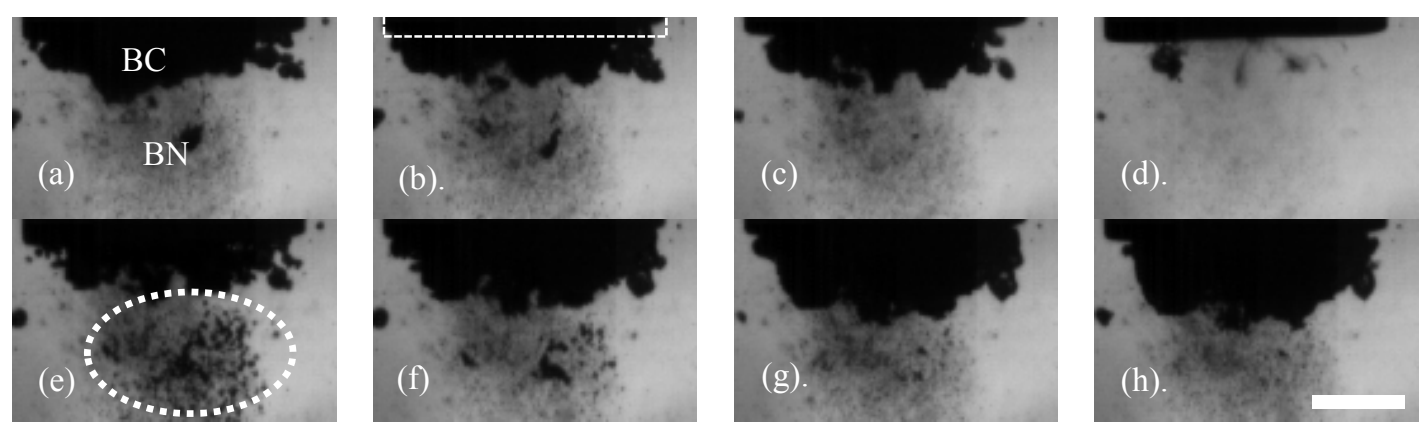

Figure 7 
Birkin et al.

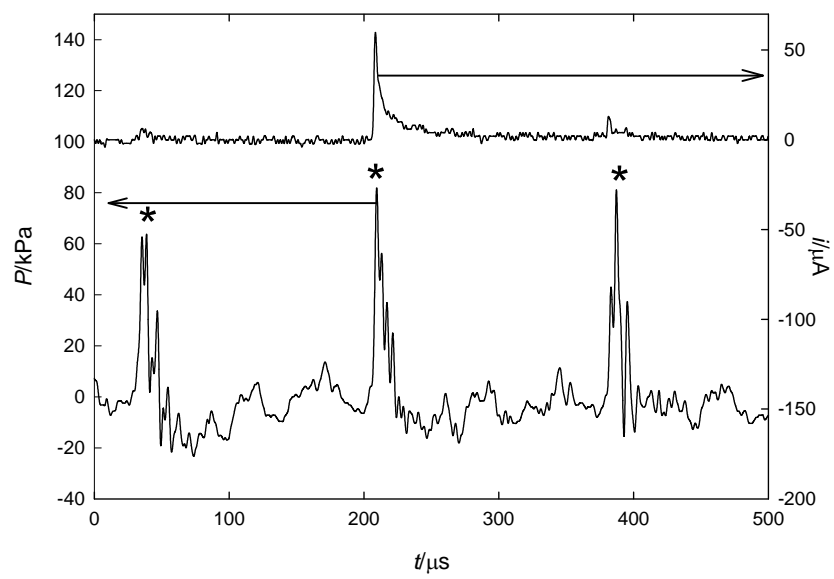

Figure 8 
Birkin et al.

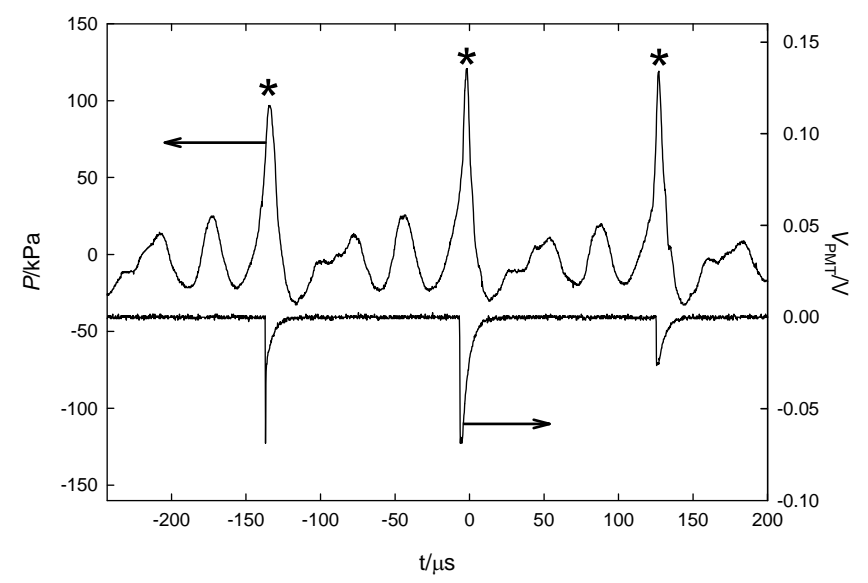

Figure 9 